\title{
Methylation and molecular profiles of ependymoma: Influence of patient age and tumor anatomic location
}

\author{
HWA JIN $\mathrm{CHO}^{1 *}$, HA YOUNG PARK ${ }^{1 *}$, KWANGSOO KIM $^{2}$, HEEJOON CHAE $^{3}$, SUN HA PAEK $^{4}$, \\ SEUNG-KI KIM ${ }^{4}$, CHUL-KEE PARK ${ }^{4}$, SEUNG-HONG CHOI ${ }^{5}$ and SUNG-HYE PARK ${ }^{6}$
}

\author{
${ }^{1}$ Department of Pathology, Inje University Busan Paik Hospital, Busan 47392; ${ }^{2}$ Division of Clinical Bioinformatics, \\ Biomedical Research Institute, Seoul National University Hospital, Seoul 03080; ${ }^{3}$ Division of Computer Science, \\ Sookmyung Women's University, Seoul 04310; Departments of ${ }^{4}$ Neurosurgery, ${ }^{5}$ Radiology and ${ }^{6}$ Pathology, \\ Seoul National University Hospital, Seoul National University College of Medicine, Seoul 03080, Republic of Korea
}

Received June 10, 2020; Accepted February 5, 2021

DOI: $10.3892 / \mathrm{mco} .2021 .2250$

\begin{abstract}
Ependymomas are tumors of the central nervous system that can occur in patients of all ages. Guidelines from the World Health Organization (WHO) for the grading of ependymomas consider patient age, tumor resection range, tumor location and histopathological grade. However, recent studies have suggested that a greater focus on both tumor location and patient age in terms of transcriptomic, genetic, and epigenetic analyses may provide a more accurate assessment of clinical prognosis than the grading system proposed by WHO guidelines. The current study identified the differences and similarities in ependymoma characteristics using three different molecular analyses and methylation arrays. Primary intracranial ependymoma tissues were obtained from 13 Korean patients ( 9 adults and 4 children), after which whole-exome sequencing (WES), ion-proton comprehensive cancer panel (CCP) analysis, RNA sequencing, and Infinium HumanMethylation450 BeadChip array analysis was performed. Somatic mutations, copy number variations, and fusion genes were identified. It was observed that the methylation status and differentially expressed genes were
\end{abstract}

Correspondence to: Professor Sung-Hye Park, Department of Pathology, Seoul National University Hospital, Seoul National University College of Medicine, 103 Daehak-ro, Jongno-gu, Seoul 03080, Republic of Korea

E-mail:shparknp@snu.ac.kr

*Contributed equally

Abbreviations: CCP, comprehensive cancer panel; CNS, central nervous system; GO, gene ontology; MAF, minor allele frequency; nsSNV, non-synonymous SNV; PF, posterior fossa; PFA, PF group A; PFB, PF group B; RELA, V-rel reticuloendotheliosis viral oncogene homolog A; SNV, single-nucleotide variant; ST, supratentorial; WES, whole-exome sequencing; WHO, World Health Organization

Key words: ependymoma, DNA methylation, molecular analysis, classification, next generation sequencing significantly different according to tumor location and patient age. Several novel gene fusions and somatic mutations were identified, including a yes-associated protein 1 fusion mutation in a child with a good prognosis. Moreover, the methylation microarray revealed that genes associated with neurogenesis and neuron differentiation were hypermethylated in the adult group, whereas genes in the homeobox gene family were hypermethylated in the supratentorial (ST) group. The results confirmed the existence of significantly differentially expressed tumor-specific genes based on tumor location and patient age. These results provided valuable insight into the epigenetic and genetic profiles of intracranial ependymomas and uncovered potential strategies for the identification of location- and age-based ependymoma-related prognostic factors.

\section{Introduction}

Ependymomas can develop at all ages. However, their incidence is greatly affected by location (1), with $90 \%$ of tumors in pediatric cases occurring intracranially, and, particularly, 70\% occurring in the posterior fossa (PF) (2). Supratentorial (ST) tumors affect both pediatric and adult patients (1); in adults, PF and spinal ependymomas develop with almost the same frequency (3).

The classification of tumors of the central nervous system (CNS), as per the World Health Organization (WHO) guidelines, delineates four important prognostic factors: Patient age, the extent of resection, location, and histopathological grade (1). Pediatric cases tend to be more severe because ependymomas commonly occur in the PF in children, whereas those in adults are more frequently found in the spinal cord (1). Of note, a multi-institutional retrospective analysis of patients with pediatric ependymomas revealed that anaplastic histopathological features and incomplete tumor resection led to a poor outcome in patients younger than 3 years of age (4).

Molecular genetic studies have also revealed distinct features associated with ependymoma subgroups. Intracranial and spinal ependymomas showed mutually exclusive gene mutations (5). Moreover, differential gene expression patterns among different CNS regions were recently identified (6). In addition, Taylor et al (6) found distinct patterns of gene 
expression and chromosome alterations among distinct ependymoma subsets that correlated well with the anatomic location of the tumor, but not with the clinical parameters or the tumor histological grade. Of note, ependymomas recapitulate the gene expression profiles of regionally specified radial glial cells, explaining the clinical heterogeneity among histologically similar tumors (6). This suggests that ependymoma treatments should target the appropriate cell-signaling pathways based on the tumor location.

Mack et al (7) studied epigenomic alterations in PF ependymomas, often associated with somatic single-nucleotide variants (SNVs); of note, these tumors are relatively rare in comparison with other organ malignancies. They divided PF ependymomas into two groups according to the patient age: PF group A (PFA) and PF group B (PFB) with PFA predominantly found in infants and associated with a poor prognosis even in the context of highly aggressive therapies, and PFB occurring in older children and adults, exhibiting a more favorable prognosis. PFA and PFB represent two very distinct molecular subgroups based on the unsupervised clustering of CpG-methylation sites, with certain genes, including CRIP1, $C Y P 26 C 1$, and $P K P 1$, exhibiting increased $\mathrm{CpG}$ methylation in most PFA tumors.

Therefore, subgroups classified according to tumor location and patient age are associated with distinct transcriptomic, genetic, epigenetic, and clinical features; in fact, these classifications could be more informative for prognostic prediction than the WHO grading alone (1). Via the confirmation of the correct grouping parameters and the selection of the corresponding genes in each group, clinicians might be able to identify the major risk groups for recurrence and identify patients most suitable for adjuvant therapy. Indeed, Pajtler et al (8) assessed 500 cases of ependymal tumors, including cases of subependymoma and myxopapillary ependymoma, that were found across all CNS compartments, age groups, and histological grades. Through DNA-methylation profiling, they identified nine molecular subgroups, with the PFA and V-rel reticuloendotheliosis viral oncogene homolog A (RELA)-positive ependymoma groups exhibiting a very poor prognosis, indicating that risk stratification by molecular subgrouping is superior to histologic grading.

Here, we analyzed the genomic and epigenomic alterations in Korean patients with intracranial ependymomas using three different methods of molecular analysis and Illumina $450 \mathrm{~K}$ methylation arrays. We attempted to identify differences and similarities in ependymoma characteristics according to each method. Our findings may support the development of an accurate classification system based on molecular subgroups to facilitate the prognosis and promote the use of personalized medicine approaches.

\section{Materials and methods}

Study subjects and experimental design. Fresh-frozen samples were collected from 13 patients who underwent ependymoma resection surgery at the Seoul National University Hospital (Seoul, South Korea): Nine adults and four children younger than 7 years of age. The female-to-male ratio was 8:5. Tumors in nine patients were located in the PF, whereas the four other patients had ST ependymomas; no cases of spinal ependymomas were identified in the cohort. Through a pathological review, six tumors were diagnosed as grade II and seven were diagnosed as grade III anaplastic ependymoma in accordance with the WHO guidelines for the classification of CNS tumors (1). The following identification criteria were further used to classify the tumors: Increased cellularity, frequent mitosis, abundant necrosis, and microvascular proliferation. High mitotic activity was defined when at least five mitotic figures were detected based on the results of a European clinical trial (9). Ki-67 immunohistochemical staining was also performed on all samples to assess the proliferation index of the tumor using an image analysis system (ScanScope XT; Aperio).

Ependymomas in two adults and three children were recurrent, with one resulting in death. The four surviving patients harbored grade-III tumors, all of which were located in the PF.

Of the 13 samples, some specimens did not pass DNA or RNA quality tests and showed insufficient DNA or RNA concentrations. Of note, experiments were only conducted with specimens that passed quality checks: 12 were used for methylation profiling, five were used for WES, seven were applied for targeted sequencing using an ion-proton CCP, and five were subjected to RNA sequencing.

\section{DNA methylation}

DNA extraction and preparation. Genomic DNA ( $\geq 500 \mathrm{ng}$ ) was isolated from all tissues. Bisulfite conversion of genomic DNA was achieved using the EZ DNA Methylation-Gold Kit (Zymo Research Corporation). Briefly, bisulfite treatment changes unmethylated cytosine nucleotides to thymidines, whereas methylated cytosines remain unchanged. This difference allows for the detection of C/T nucleotide polymorphisms at each $\mathrm{CpG}$ site. Genomic DNA was sent to Macrogen for hybridization on Illumina $450 \mathrm{~K}$ methylation arrays (Illumina, Inc.). The Illumina Infinium human methylation 450 BeadChip kit (Illumina, Inc.) was used to evaluate the coverage of $\mathrm{CpG}$ sites throughout the genetic regions according to the manufacturer's instructions. BeadChips were scanned with an Illumina iScan apparatus (Illumina, Inc.).

Data processing and analysis. Following image analysis, data processing was performed using the Illumina GenomeStudio software version 2011.1 (Methylation Module v1.9.0; Illumina, Inc.) and the R package, version 3.0.2 (http://www.r-progect.org). Data preprocessing and background correction were performed using the R library and dye-bias equalization. The detected $\mathrm{CpGs}$ were filtered (per sample) according to the P-value. On average, between 485,139.3 and 485,262.6 CpGs were detected with a cut-off value of $\mathrm{P}<0.05$. Next, the beta mixture quantile in the $\mathrm{R}$ package was used to correct any probe-design bias. To perform data transformation, the $\beta$-value, M-value, and $\delta$-mean were calculated. $\beta$-value represents the ratio of methylated probe intensity to the sum of methylated and unmethylated probe intensities. M-value is calculated as the $\log _{2}$-ratio of the intensities of the methylated probes vs. those of the unmethylated probes $(10)$. $\delta$-mean represents the difference between the average $\beta$-value of the experimental results and that of the controls. The odds ratio was calculated via the transformation of the $\delta$-mean into the M-value and the 
measurement of the ratio between the unmethylated intensity and methylated intensity for the test sample against that of a control. Fold expression changes were calculated as the ratio of methylation rates between test and control specimens.

Hierarchical clustering analyses. Using the M-values from significant data, 16,312 CpGs in different age groups and 5,034 CpGs in different grade groups were obtained with cut-off values of $\mathrm{P}<0.05$ and a $\delta$-mean $>0.2$. Among them, $500 \mathrm{CpGs}$ were randomly selected. A heatmap of hierarchical clustering was plotted based on the distance similarity for samples and CpGs according to the Euclidean distance and complete linkage.

Assessment of differential methylation patterns. To determine the difference in DNA methylation profiles among samples, especially according to patient and clinical attributes such as age and tumor location, two basic approaches were adopted. First, the methylation status of $\mathrm{CpG}$ sites in the regulatory regions of target genes was classified: Hyper- or hypomethylated regions were defined depending on their methylation levels- $\beta$-values of $>0.7$ and $<0.3$ were adopted as the cut-offs for hypermethylation and hypomethylation, respectively. For the second approach, we determined the differences in the absolute DNA methylation status between samples, regardless of their classification as hypermethylated or hypomethylated. For instance, if the $\beta$-value from a pediatric patient's sample was 0 and that of an adult patient's sample was 0.6 , the first approach would not detect this difference as statistically significant, despite the difference of 0.6 in the $\beta$-value. In some cases, the differences obtained using the second method were greater than those using the first approach. Therefore, to capture the absolute differences in DNA methylation, the $\beta$-values between samples for the same $\mathrm{CpG}$ sites were subtracted from both the age and tumor location groups. In this approach, a $\beta$-value cut-off of 0.4 was set to identify significant differences between groups.

Gene enrichment and functional annotation analyses. Through these two analysis approaches, we successfully extracted target genes with a significant difference in DNA methylation in their regulatory regions (especially within the promoter and gene body regions). To examine gene ontology (GO), gene set analysis was performed using ToppGene (https://toppgene.cchmc.org/), a gene list enrichment analysis and candidate gene prioritization system based on functional annotations and protein interaction networks (11).

\section{WES}

DNA sequencing. DNA from five resected fresh-frozen ependymoma samples was extracted using the EZ DNA methylation gold kit (Zymo Research Corporation). DNA extracted from blood samples of the same patients was used as germline control. To enrich the coding regions, the SureSelect target-enrichment system capture process (Agilent Technologies, Inc.) was used. Sequence reads were generated using the Illumina HiSeq 2500 platform (12).

Data analysis. Raw sequence reads were mapped against the hg19 reference genome using a Burrows-Wheeler aligner (13).
After creating a BAM file, PCR duplicates were removed using Picard (https://broadinstitute.github.io/picard/index. html). Insertion and deletion (indel) realignment and base quality score recalibration were performed using the Genome Analysis Tool Kit $(14,15)$. The VarScan2 software was used to detect somatic SNVs and small indels (16). ANNOVAR was used to annotate variants. To identify confident and rare somatic variants in the coding regions and splice sites, the following filtering criteria were applied: i) tumor total allele count $\geq 10$, tumor altered allele count $\geq 3$, and normal altered allele count $=0$; ii) rare variants were based on a minor allele frequency (MAF) $<0.5 \%$ in the Exome Aggregation Consortium Version 0.3 (ExAC03; http://exac.broadinstitute. org) (17).

Comprehensive cancer panel. To identify the mutation status in 409 cancer-related genes, targeted sequencing of seven tumor samples was performed using the Ion AmpliSeq Comprehensive Cancer Panel (CCP; Life Technologies; Thermo Fisher Scientific, Inc.). Mutations on the list showing non-synonymous SNVs and indels in coding regions with an altered allele frequency $\geq 0.1$, and rare variants based on a MAF $<0.5 \%$ in the Exome Aggregation Consortium Version 0.3 were used for further analysis.

RNA sequencing. Using the Illumina TruSeq RNA sample preparation kit (Illumina, Inc.), mRNA was converted into a template library for subsequent cluster generation. Purified mRNA was fragmented, and reversely transcribed into cDNA. DNA polymerase I and RNase $\mathrm{H}$ were then used to construct second-strand cDNA. cDNA fragments had an additional adenines added to their 3 '-end and the adapters were then ligated. Products were then enriched by PCR and HiSeq 2000 (Illumina, Inc.) was used for bridged-amplification reactions and imaging. Images of single-base extensions at a specific cluster were generated.

Sequence quality checks were performed using Fast(v0.10.0; www.bioinformatics.babraham.ac.uk/projects/fastqc/). The alignment was performed via TopHat (v1.3.3; https://ccb. jhu.edu/software/tophat/index.shtml), a fast splice-junction mapper for RNA-seq reads, with hg19 (18). Cufflinks (v2.0.2; http://cole-trapnell-lab.github.io/cufflinks/) was used for transcript assembly and to test for the differential expression and regulation of RNA-seq samples (19). Gene expression was measured in terms of fragments per kilobase of transcript per million mapped reads.

To detect SNVs and make annotations, SAMtools was used; alignments were manipulated in the SAM format (http://samtools.sourceforge.net/cns0.shtml). ANNOVAR (http://www.openbioinformatics.org/annovar/) and DeFuse (v0.4.3; http://sourceforge.net) were used to annotate functional genetic variants and to discover fusion genes, respectively.

Differentially expressed genes were detected via the comparison of the mean of the original value for each group against the mean of the Z-score for each group. Genes exhibiting the largest differences in expression were analyzed and validated via a literature search for their relevance to ependymoma, and included in the human disease database (MalaCards; http://malacards.org/card/ependymoma?limit; 
Table I. Summary of the clinicopathologic and genomic characteristics of the patients included in the present study.

\begin{tabular}{lccccccccccccc}
\hline Variable & P1 & P2 & P3 & P4 & P5 & P6 & P7 & P8 & P9 & P10 & P11 & P12 & P13 \\
\hline Age (years) & 32 & 48 & 53 & 48 & 25 & 41 & 29 & 48 & 6 & 2 & 3 & 3 & 38 \\
Sex & F & M & F & F & F & M & F & M & F & M & M & F & F \\
Tumor location & ST & ST & PF & PF & PF & PF & PF & ST & ST & PF & PF & PF & PF \\
Recurrence & N & N & N & N & N & N & Y & Y & N & Y & Y & Y & N \\
Death & N & N & N & N & N & N & N & N & N & N & Y & N & N \\
Histologic grade & GII & GII & GII & GII & GIII & GIII & GIII & GIII & GII & GIII & GIII & GIII & GII \\
No. of SNVs by WES & NA & NA & 9 & NA & 9 & 12 & NA & NA & 3 & NA & NA & 9 & NA \\
No. of fusion genes by & NA & NA & 2 & NA & 3 & 0 & 3 & NA & 1 & NA & NA & NA & NA \\
RNA sequencing & & & & & & & & & & & & & \\
CCP & A & A & NA & A & NA & NA & NA & A & NA & A & A & NA & A \\
Methylation & A & A & A & A & A & A & A & A & A & A & A & A & NA \\
\hline
\end{tabular}

P, patient; F, female; M, male; ST, supratentorial; PF, posterior fossa; N, No; Y, Yes; G, grade; SNVs, single nucleotide variants; WES, whole exome sequencing; NA, not applicable; A, analyzed; CCP, comprehensive cancer panel.

[MaladiesGenes]=128\#related_genes). The Student's $t$-test was used to identify expression differences.

\section{Results}

Histopathological features. Of the thirteen samples, six were classified as grade II ependymoma (low-grade; LG) and seven were classified as grade III anaplastic ependymoma (high-grade; HG). Increased cellularity was observed in five grade II ependymomas and in all grade III ependymomas. The mitotic count ranged from 0 to 4 (mean, 2.5) in grade II, and from 11 to 26 (mean, 19.0) in grade III ependymoma samples. Microvascular proliferation was observed in only one grade III ependymoma. Abundant necrosis was observed in four cases of grade III ependymoma and focal necrosis was observed in three cases of grade II ependymoma. The Ki-67 staining index ranged from $<1$ to $11.64 \%$ (mean, $5.0 \%$ ) in grade II ependymomas, and from 4.48 to $32.97 \%$ (mean, $16.2 \%$ ) in grade III ependymomas. The clinicopathological information of the patients, the experimental design and the summarized genomic characteristics are shown in Tables I and SI.

\section{DNA methylation}

Hierarchical clustering. Significant differences in the methylation rates associated with patient age, tumor grade, and location were assessed via hierarchical clustering. Hierarchical clustering according to age respected the significance criterion $(\mathrm{P}<0.05)$ with an absolute $\delta$-mean value of $>0.2$ using 500 randomly selected CpGs (Fig. 1A). In each clustered age-group, unsupervised clustering by anatomic location (ST and PF) was prominently observed. On the other hand, other factors, including grade and prognosis (recurrence), were not categorized in the same groups.

Hierarchical clustering by grade also showed clustering by location in each grade group (Fig. 1B). The HG group was separated into the ST group, including one patient, and the PF group, including six patients. Children and adults were clustered as separate groups for HG tumors in the PF. Overall, the methylation patterns showed meaningful differences with respect to both tumor location and patient age.

Methylation profile based on age and location. We next counted the number of genes exhibiting significant differences in methylation based on patient age and tumor location. Many genes were identified to have a $\beta$-value difference of $>0.4$ between adults and children (Table SII); of note, 66 genes were included in the adult-hypomethylated group and 425 genes were included in the adult-hypermethylated group. Three of these genes (PDE4C, HOXA9, and TBX5) in the adult-hypermethylated and child-hypomethylated groups showed the most substantial age-related differences, in line with data previously reported by Koch and Wagner (20) in an epigenetic study. These genes showed higher methylation in adults vs. children. Thus, using the absolute $\beta$-value is preferred over using the difference in the methylation rate to determine target genes in each age or location group due to the potential interference of age-related methylation. Of note, we used $\beta$-values of 0.7 and 0.3 as the hypermethylation and hypomethylation cut-off values, respectively, because we could not obtain significant data using the cutoff points of 0.8 or 0.2 proposed by Rogers et al (2).

GO analysis revealed that a high proportion of genes displaying hypermethylation in adults was included in GO sets; 20 of the 1,638 genes associated with neurogenesis were identified in our samples, as well as 16 of the 1,405 genes associated with neuron differentiation (Fig. 2A). Many genes belonging to the HOX family were observed in several pathways. We also found many genes associated with the development of multicellular organisms (neurogenesis and embryo development) in the biological process category of GO enrichment analysis (Fig. 2B). Moreover, the analysis of Reactome pathways revealed that genes related to $G$ protein-coupled receptors and amine compound solute carrier transporters were enriched (Fig. 2C). Importantly, these pathways are crucial targets for drug development.

Moreover, many genes showed $\beta$-values $>0.4$ between the PF and ST groups (Table SIII). Sixty-one genes were included in the PF-hypomethylated group and fourteen genes 
A

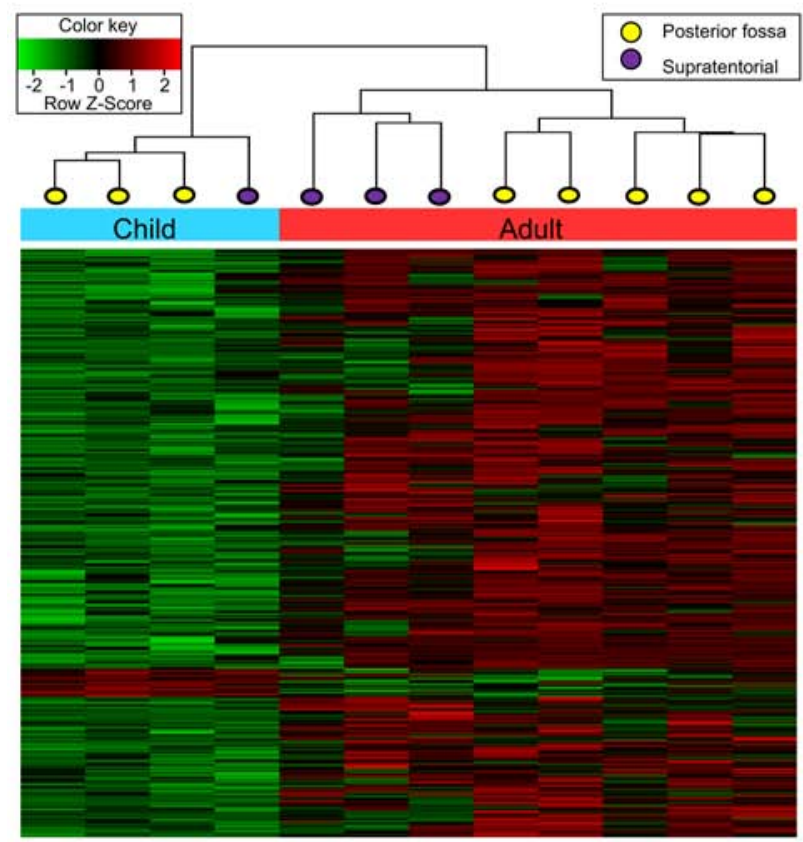

B

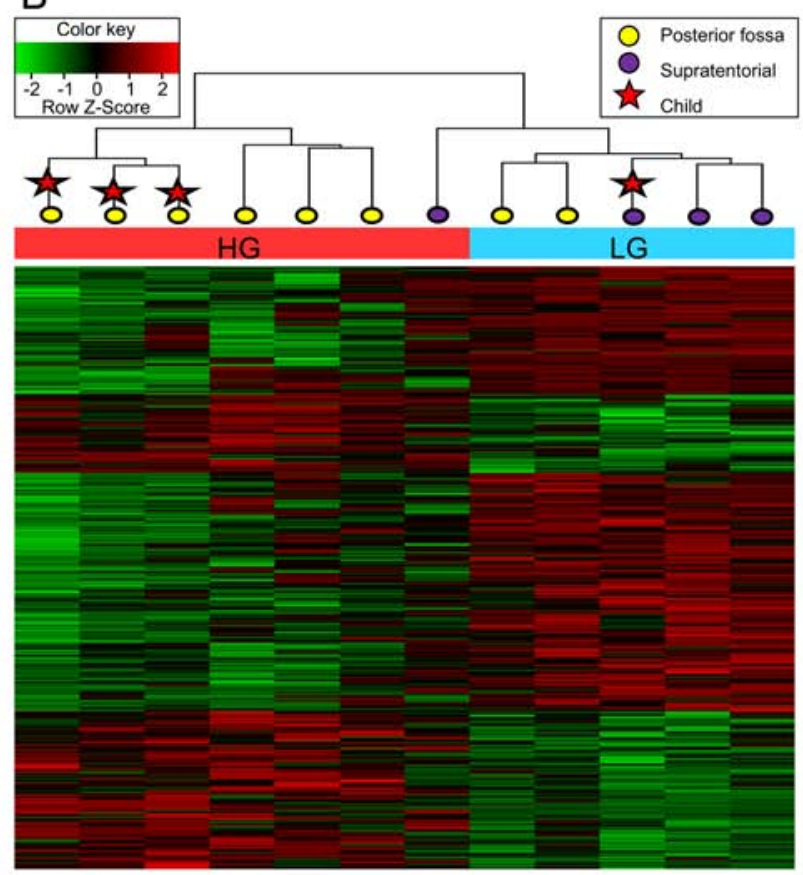

Figure 1. Two-way hierarchical clustering dendrogram of methylation patterns between adults and children. (A) and LG and (B) HG tumors. (A) unsupervised hierarchical clustering by location (supratentorial and PF) was prominently observed via supervised hierarchical clustering by age using 500 randomly selected CpGs. (B) Clustering by location in each grade group was observed via supervised hierarchical clustering by grade. In the $\mathrm{HG}(\mathrm{n}=7)$ and $\mathrm{PF}(\mathrm{n}=6)$ groups, clustering according to both age and location was observed. LG, low grade; HG, high grade; PF, posterior fossa.

A

\begin{tabular}{|c|c|c|c|c|c|c|c|c|c|c|c|c|c|}
\hline $\begin{array}{l}\text { ㄷㅇㄹ } \\
\text { 담 } \\
\text { L }\end{array}$ & 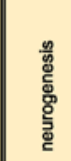 & 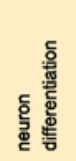 & 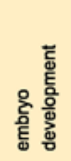 & 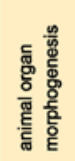 & 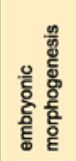 & 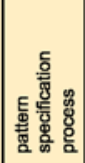 & 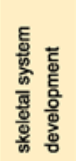 & 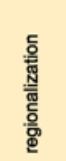 & 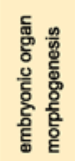 & 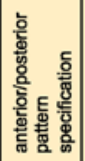 & 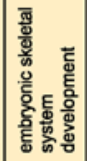 & 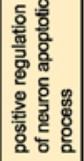 & 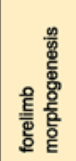 \\
\hline \multicolumn{14}{|l|}{ HOXA7 } \\
\hline \multicolumn{14}{|l|}{ HOXA9 } \\
\hline \multicolumn{14}{|l|}{ HOXB6 } \\
\hline \multicolumn{14}{|l|}{ HOXC4 } \\
\hline \multicolumn{14}{|l|}{ HOXC5 } \\
\hline \multirow{2}{*}{\multicolumn{14}{|c|}{$\begin{array}{l}\text { HOXC6 } \\
\text { HOXC8 }\end{array}$}} \\
\hline & & & & & & & & & & & & & \\
\hline \multicolumn{14}{|l|}{ HOXD1 } \\
\hline \multicolumn{14}{|l|}{ NEUROG1 } \\
\hline \multicolumn{14}{|l|}{$\mathrm{NKX} 2-5$} \\
\hline \multicolumn{14}{|l|}{$\begin{array}{l}\text { GABRB3 } \\
\text { KCNMA1 }\end{array}$} \\
\hline \multicolumn{14}{|l|}{ KCNMA1 } \\
\hline NTNG1 & & & & & & & & & & & & & \\
\hline STXBP5 & & & & & & & & & & & & & \\
\hline \multicolumn{14}{|l|}{ TNN } \\
\hline \multicolumn{14}{|l|}{ UNC5A } \\
\hline \multicolumn{14}{|l|}{ CDKN2C } \\
\hline \multicolumn{14}{|l|}{ PRDM13 } \\
\hline \multicolumn{14}{|l|}{ KCNMA1 } \\
\hline \multicolumn{14}{|l|}{ ASPH } \\
\hline \multicolumn{14}{|l|}{ LUM } \\
\hline \multicolumn{14}{|l|}{ CDC73 } \\
\hline \multirow{2}{*}{\multicolumn{14}{|c|}{$\begin{array}{c}\text { THRB } \\
\text { SOSTDC1 }\end{array}$}} \\
\hline & & & & & & & & & & & & & \\
\hline \multicolumn{14}{|l|}{ TCF21 } \\
\hline \multicolumn{14}{|l|}{ LUM } \\
\hline \multicolumn{14}{|l|}{ NR3C1 } \\
\hline EPB41L3 & & & & & & & & & & & & & \\
\hline TFAP2A & & & & & & & & & & & & & \\
\hline TFAP2B & & & & & & & & & & & & & \\
\hline GFI1 & & & & & & & & & & & & & \\
\hline EXOC4 & & & & & & & & & & & & & \\
\hline WNT3 & & & & & & & & & & & & & \\
\hline p-value & $2.03 \mathrm{E}$ & $1.30 \mathrm{E}-\mathrm{C}$ & 4.64E-05 & 3.95 & $12 \mathrm{E}-05$ & & $9.52 \mathrm{E}-$ & 1.80 & $2 E-0$ & $3.50 \mathrm{E}-\mathrm{C}$ & $1.35 \mathrm{E}-0$ & 1.75 & 3.25E- \\
\hline FDR q-value & 6. & 25 & $1.08 \mathrm{E}-02$ & 2 & 24 & 1.5 & 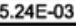 & 0.16 & 1.1 & 2.1 & $1.58 \mathrm{E}-03$ & $3.15 \mathrm{t}$ & \\
\hline
\end{tabular}

B

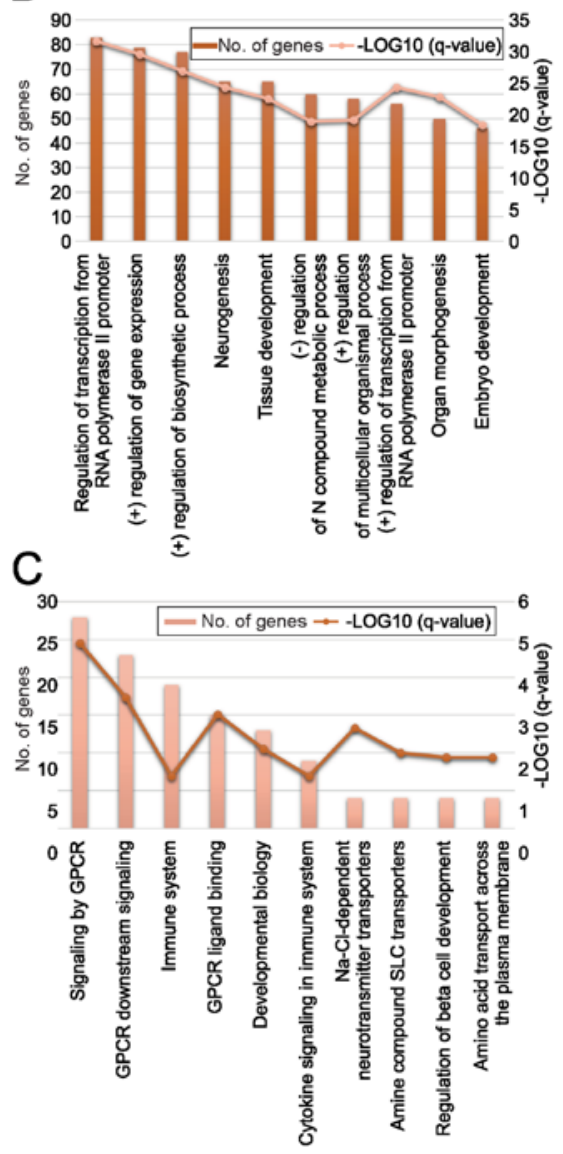

Figure 2. Functional enrichment of hypermethylated genes in the adult group. (A) Genes that were hypermethylated in the adult group were sorted per gene ontology functions. (B) The top 10 gene sets in the biological process category of gene ontology enrichment analysis. (C) The top 10 reactome pathways enriched in hypermethylated genes. GPCR, G-protein coupled receptor; SLC, solute carrier. 


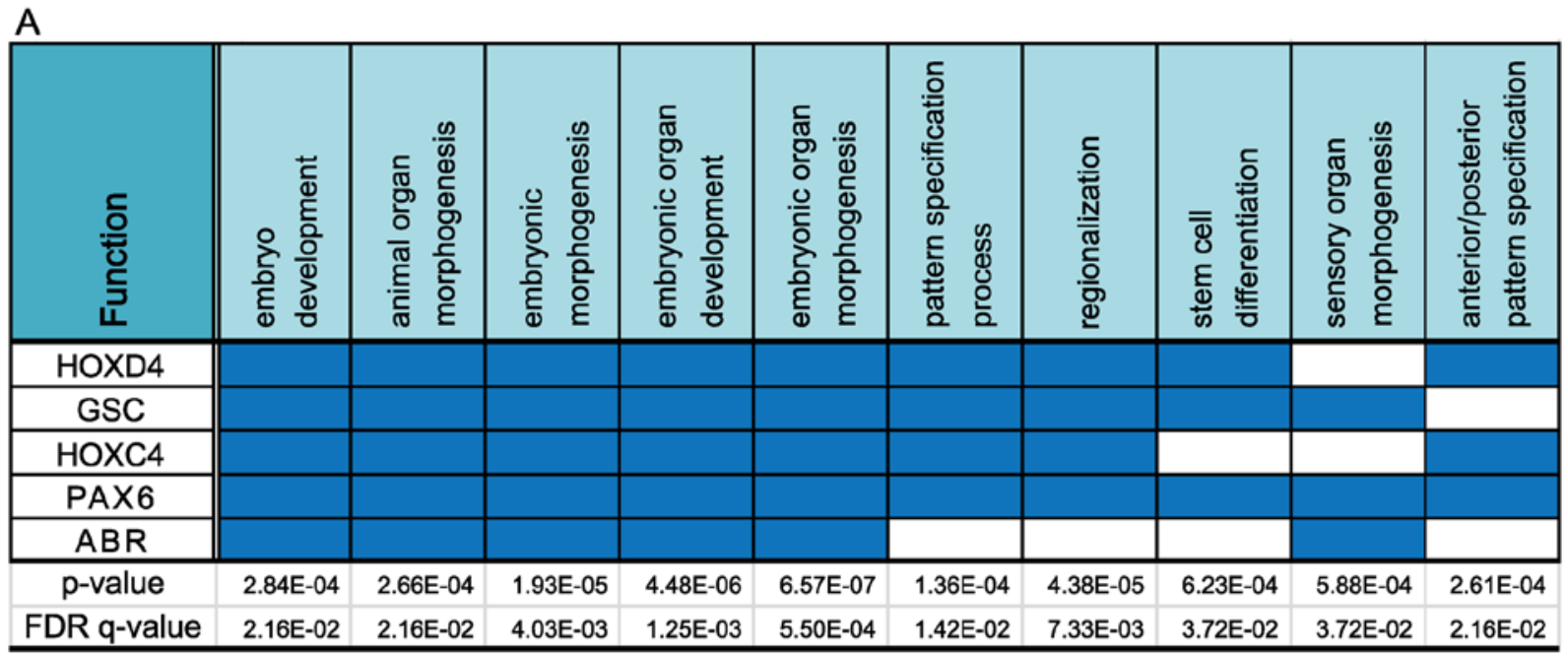

B

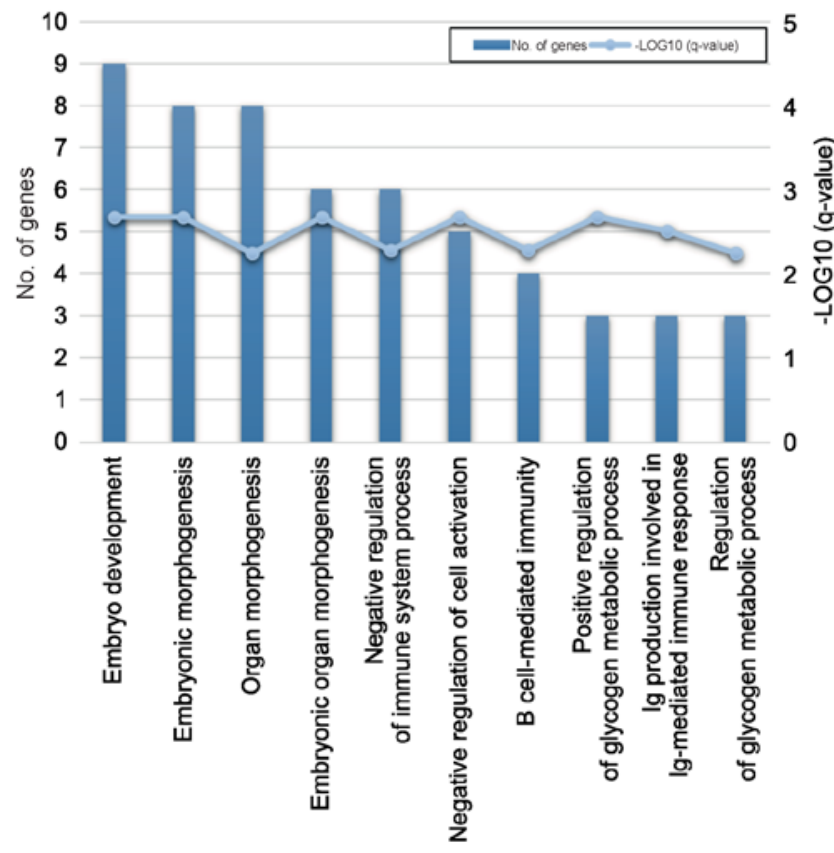

C

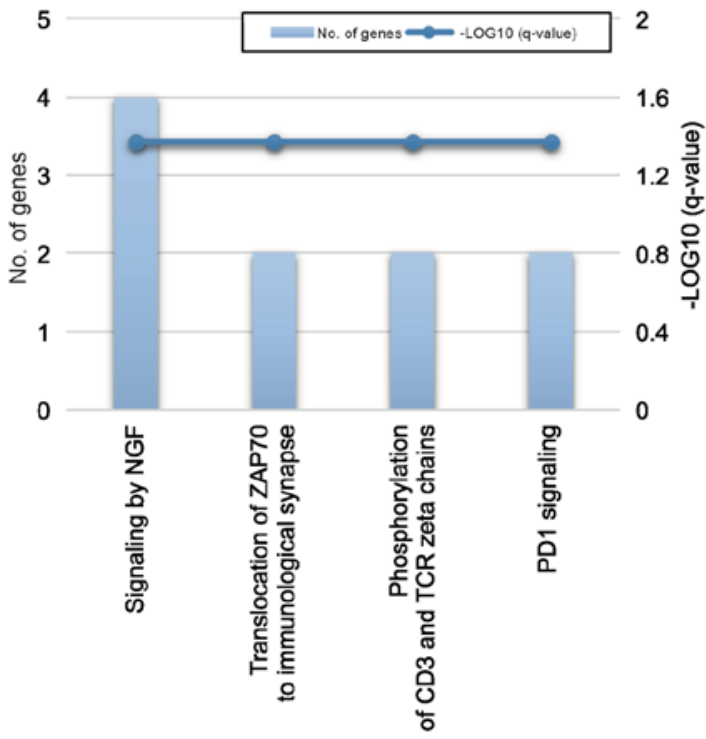

Figure 3. Functional enrichment of hypermethylated genes in the ST group. (A) Genes that were hypermethylated in the ST group sorted per gene ontology functions. (B) The top 10 gene sets in the biological process category of gene ontology enrichment analysis. (C) Reactome pathways enriched in hypermethylated genes. ST, supratentorial; NGF, nerve growth factor; ZAP70, zeta-chain-associated protein kinase 70; TCR, T-cell receptor; PD1, programmed death 1.

were included in the PF-hypermethylated group. However, no significant genes were detected in the PF-hypermethylated group, as per the $\mathrm{GO}$ analysis.

GO pathway analysis revealed that five genes (HOXD4, $G S C, H O X C 4, P A X 6$, and $A B R$ ) overlapped in a single biological process category (Fig. 3A). Among them, HOXD4, HOXC4, and $P A X 6$, identified in the PF-hypomethylated/ST-hypermethylated group, were associated with hindbrain development during early embryogenesis. Moreover, many genes associated with the development of multicellular organisms (embryo development and embryonic morphogenesis) and the development of embryonic organs (organ morphogenesis and embryonic organ morphogenesis) were identified in the biological processes' categorization of the GO enrichment analysis (Fig. 3B). Finally, nerve growth factor signaling-associated genes were enriched, as per the Reactome pathway analysis (Fig. 3C).
WES. We performed WES for five paired tumor-normal tissue samples, including two LG tumors and three HG tumors. Three patients were adults and two were children. Tumors of four of these patients were located in the PF and the tumor of one patient was located in the ST.

Fifty-eight non-synonymous SNVs (nsSNVs) were identified in the five paired samples, ranging from 7-14 nsSNVs per sample. The median number of nsSNVs per Megabase was 0.26 in adults and 0.20 in children. After filtering for rare mutations, each sample showed 3-12 nsSNVs. Interestingly, the mean number of rare nsSNVs in children was smaller than that in adults, although the difference was not statistically significant (Table SIV).

In four samples with both WES and RNA sequencing data available, the SNVs were checked to determine whether they were transcribed or edited in the context of RNA sequencing. 
Table II. Fusion genes identified using RNA sequencing.

\begin{tabular}{|c|c|c|c|c|c|c|c|c|}
\hline $\begin{array}{l}\text { Patient } \\
\text { ID }\end{array}$ & Gene name 1 & Gene name 2 & $\begin{array}{l}\text { Split read } \\
\text { count }\end{array}$ & $\begin{array}{l}\text { Span read } \\
\text { count }\end{array}$ & $\begin{array}{c}\text { Gene } 1 \\
\text { chromosome }\end{array}$ & $\begin{array}{c}\text { Gene } 2 \\
\text { chromosome }\end{array}$ & $\begin{array}{c}\text { Genomic break } \\
\text { position } 1\end{array}$ & $\begin{array}{c}\text { Genomic break } \\
\text { position } 2\end{array}$ \\
\hline \multirow[t]{2}{*}{ P3 } & PDE4DIP & AL592284.1 & 10 & 6 & 1 & 1 & 144952201 & 144508284 \\
\hline & PTMS & PCBD2 & 5 & 8 & 12 & 5 & 6879325 & 134260382 \\
\hline \multirow[t]{3}{*}{ P5 } & HNRNPA2B1 & RGPD2 & 6 & 5 & 7 & 2 & 26236085 & 88124575 \\
\hline & ZFP36L1 & RGPD2 & 4 & 5 & 14 & 2 & 69257549 & 88124733 \\
\hline & EEF1G & PCBD2 & 6 & 5 & 11 & 5 & 62339358 & 134260418 \\
\hline \multirow[t]{3}{*}{ P7 } & RAB6C & OR4Q3 & 7 & 5 & 2 & 14 & 130737953 & 20134468 \\
\hline & GFAP & PCBD2 & 15 & 15 & 17 & 5 & 42991130 & 134260432 \\
\hline & IRAK2 & NDFIP1 & 1 & 5 & 3 & 5 & 10282302 & 141532120 \\
\hline P9 & YAP1 & MAMLD1 & 13 & 6 & 11 & $\mathrm{X}$ & 102076805 & 149638017 \\
\hline
\end{tabular}

PDE4DIP, phosphodiesterase 4D interacting protein; PTMS, parathymosin; PCBD2, pterin-4-alpha-carbinolamine dehydratase 2; HNRNPA2B1, heterogeneous nuclear ribonucleoprotein A2/B1; RGPD2, RAN binding protein 2-like and GRIP domain containing 2; ZFP36L1, zinc finger protein 36 homolog like 1; EEF1G, eukaryotic translation elongation factor 1 gamma; RAB6C, Rab protein family 6C; OR4Q3, olfactory receptor family 4 subfamily Q; GFAP, glial fibrillary acidic protein; IRAK2, interleukin-1 receptor-associated kinase 2; NDFIP1, Nedd4 family interacting protein 1; YAP1, yes-associated protein 1; MAMLD1, mastermind-like domain containing 1; P, patient.

SNVs identified in seven genes (HARS2, SPSB3, BAZ2B, TRIO, SNX13, PLXNA4, and PARP12) in WES were indeed transcribed, as per the RNA sequencing results (Table SV). In contrast, no case of editing was identified.

In the copy number variation (CNV) analysis, patient 3 (53-year-old female; LG and PF) showed insertions in chromosomes $1,4,5,7,8,9,12,14,15,18$, and 19. Patient 5 (25-year-old female; HG and PF) showed gains in chromosomes 12, 19 and 20, and losses in chromosomes 3, 6, 13, 16, 17, 21 and 22. Patient 6 (41-year-old male; HG and PF) showed an increased copy number in chromosome 1q and losses in chromosomes 3 , 5, 17, 21 and 22. Patient 9 (6-year-old female; LG and ST) showed copy loss in chromosome 11, and patient 12 (3-year-old female; HG and PF) showed copy gain in chromosome 19. In summary, three of four PF cases showed a copy gain in chromosome 19, which is a rare finding. Moreover, children showed fewer CNVs than adults, as expected. Interestingly, trisomy 19 ependymomas, classified as ST WHO grade-III tumors in children (21), are usually associated with a clear cell morphology, which was not evident in our cases.

Comprehensive cancer panel. To identify the mutation status of cancer-related genes, we used the Ion AmpliSeq CCP. Among the 409 oncogenes included in the panel, 249 SNVs and indels in 156 genes were identified. A few mutations were observed in all seven patients. The FGFR3, KIT, and TAF1L genes harbored nonsynonymous SNVs, whereas the KMT2C gene harbored a deletion-insertion mutation; however, none of the mutations were pathognomic. Of note, we excluded two indels that occurred in a repeat area, which is a common error of the Ion AmpliSeq platform.

Mutations in the CASC5 gene were identified in both WES and CCP analyses; however, their positions were different. Despite filtering common variants according to the population MAF database, probable germline variants were difficult to filter out without the corresponding information on paired normal samples.
RNA sequencing. The RNA sequencing results for five patients revealed the presence of multiple fusion genes (Table II), including a YAPl fusion in patient 9 (child; ST and LG), common in cases of pediatric ST ependymomas. The other fusion genes identified in three patients were novel fusion genes.

We compared the results with respect to severity ( $\mathrm{LG}, \mathrm{n}=2$ vs. $\mathrm{HG}, \mathrm{n}=3$ ), age/location (adults or $\mathrm{PF}$ area, $\mathrm{n}=4$ vs. children or ST, $n=1$ ), and sex (female, $n=4$ vs. male, $n=1$ ). Differentially expressed genes were detected via the comparison of the mean of the original value for each group against the mean of the corresponding Z-score. Genes exhibiting the largest differences in expression were analyzed; validation was further achieved via a literature search: Their relevance to ependymoma was studied and they were included in the MalaCards human disease database.

The differences in the original values associated with the RNA-seq results indicated that upregulated genes were preferentially ranked. In fact, genes such as GFAP and $S 100 B$ exhibited high levels of expression in the context of ependymoma. Therefore, they could not be used to confirm the presence of group differences. Because differences in Z-scores reveal relative differences in expression, originally small values have no significance. Therefore, the possibility of finding group-specific genes is only high when using intersecting genes.

The differences in the expression levels were the greatest between females and males in all group comparisons based on correlations. Our analysis showed that 595 genes were differentially expressed between the LG and HG groups $(\mathrm{P}<0.05)$. Of these genes, four ( $E Z R, P D P N, S H C 3$, and $P R G 2)$ were directly related to ependymoma according to the MalaCards analysis.

\section{Discussion}

Our data support the recent WHO guidelines describing ependymoma prognosis parameters using a 'cell of origin' concept, 
suggesting that distinct groups of stem cells are specific to anatomical sites, and that the different origins of the stem cells can explain the predominant locations of ependymomas detected in different age groups (6). Our methylation analysis and RNA sequencing results suggested that there may be differences in gene expression profiles between different age groups as well as anatomical locations. In addition, the hierarchical clustering of 500 randomly selected $\mathrm{CpG}$ associated with differentially expressed genes $(\mathrm{P}<0.05$ and $\Delta$ mean $)$ demonstrated prominent differences between the ST and $\mathrm{PF}$ groups in both children and adults. Although ependymoma histology cannot reveal the clinical differences corresponding to patients with ST or PF tumors, the genetic profiles implied the presence of distinct phenotypes related to tumor location.

Drugs that target $\mathrm{CpG}$ methylation or histone demethylase inhibitors are considered potential therapeutic agents for the treatment of pediatric PFA ependymomas (22). Based on our results, targeting certain genes of the $H O X$ family in adults (HOXA7, HOXA9, HOXB6, HOXC4, HOXC5, HOXC6, and HOXC8), or in ST tumors (HOXC4,HOXD4), may indeed be a useful treatment strategy. Of note, the HOX gene family plays a key role in development, providing the anterior and posterior axial coordinates for vertebrate embryos (23); e.g., the expression of $H O X A 1-2$ was associated with the hindbrain, whereas that of $H O X A 9-13$ was predominantly seen in the extremities (24).

A few $H O X$ family genes have been implicated in spinal cord ependymomas; for instance, HOXA9 (6), and HOXB5 (25) were highly expressed in spinal tumors. In fact, some $H O X$ family members, especially $H O X A 9$, are selectively expressed in spinal ependymomas (26). However, in our study, HOXA9 was hypermethylated in intracranial ependymomas, in adults. Koch and Wagner (20) reported that HOXA9 was an age-related gene in different tissues such as the dermis or cervical smear cells. Further studies may be necessary to discover the function of HOXA9 in intracranial ependymomas associated with aging.

In our epigenetics analysis using a methylation array, large differences in the methylation levels of many genes were detected between age groups. In adults, several more genes were associated with hypermethylation than with hypomethylation; these were largely related to neurogenesis and neuron differentiation (as per the GO analysis), representing a meaningful association with respect to tumor properties. Genes associated with hypomethylation in the PF group mainly included those in the $H O X$ family, which Taylor et al (6) suggested as a representative gene family that maintains the cancer stem cell phenotype in spinal ependymomas.

Molecular alterations are common in ependymoma. Therefore, accurate molecular subgrouping is necessary to predict patient outcomes and determine the best therapeutic approach. In the revised fourth edition of the WHO guidelines for CNS diseases, nine molecular groups were suggested based on a single study conducted by Pajtler et al (8). This analysis focused on the use of DNA-methylation profiling to determine ependymoma subgroups and identified two ST subgroups characterized by the fusion genes RELA and YAP1 (8). Their results also indicated molecular subgroups showing distinct copy number profiles, consistent with our findings concerning CNVs, as per WES analysis.
We compared our WES data with those reported by Korshunov et al (27), who proposed three distinct molecular stages for intracranial ependymoma, based on patterns of cytogenetic alterations in 122 primary ependymoma samples from adults and children. Group 1 ependymomas were characterized by numerous aberrations affecting whole chromosomes or chromosome arms, including gains in chromosomes $9,15 \mathrm{q}$, and/or 18, and/or a loss in chromosome 6 without a 1q gain, and/or $C D K N 2 A$ deletion. These alterations were observed in $34 \%$ of all patients and were associated with a $100 \% 5$-year overall survival rate. Of note, patients 3 and 5 in our study exhibited characteristics that would place them into this group, and these patients were disease-free for 2 years and 1 year, respectively. Group 2 ependymomas, were characterized by a largely balanced genomic profile, and were observed in $42 \%$ of patients, with 5-year overall survival rates of $78 \%$ (27). In our study, patient 9 and patient 12 could be classified into this group. Patient 9, who was diagnosed with ST/LG ependymoma showed no recurrence for 49 months. However, patient 12, a child with $\mathrm{PF} / \mathrm{HG}$ ependymoma, showed recurrence for five times, despite repeated gamma knife radiosurgery and radiotherapy. Finally, group 3 ependymomas showed copy number gains in chromosome 1q and/or homozygous $C D K N 2 A$ deletion; $25 \%$ of patients were classified in this group, exhibiting 5 -year survival rates of $32 \%$, constituting the worst overall prognosis. Patient 6 in our study was included in this group and experienced no recurrence or death over a 16-month follow-up period. Overall, these results indicate that the CNV data alone cannot be used for an accurate molecular classification, due to the potential poor reproducibility.

Compared to the methylation microarray analysis, several molecular analysis tools have failed to find sufficient evidence to support genetic features associated with a particular disease or subtype. Before filtering the WES data, the absolute number of somatic mutations per Megabase was larger in adults, possibly resulting from the presence of age-related mutations. On the other hand, after filtering, few nsSNVs were detected via WES, especially in children, compared to those reported in other malignancies, in line with a previous analysis of PF ependymoma in children (7). In addition, a few mutations were identified in the CCP analysis, and no mutations were common between the WES and CCP datasets.

We detected a few fusion genes via RNA sequencing, including a $Y A P 1$ fusion in a pediatric patient who had a tumor in the ST area and showed a good prognosis. This result is in line with the Pajtler's molecular classification (8). The other novel fusion genes identified in this study are not listed in the COSMIC database, possibly indicating that they are not functional. Another well-known fusion gene in addition to YAPl is RELA, which has been identified in about $70 \%$ of ST ependymoma cases in children (28). The C11orf95-RELA fusion is the most common structural variant found in ependymomas (8). In our RNA fusion study, only one available sample was from the ST; remarkably a $Y A P 1$ fusion was detected in that sample. However, we did not find any RELA fusion in the five ST samples, possibly due to the small sample size.

Among the genes displaying a significant difference between the adult/PF and children/ST groups with respect to both the original genetic value and Z-scores in RNA sequencing, only TIMP3 was listed in the human disease 
database MalaCards. TIMP3, which plays a role in the development of the CNS and in the proteolysis pathway, was identified as one of the candidate methylated genes re-expressed in short-term cell cultures of ependymoma samples from pediatric patients following treatment with the demethylating agent 5-aza-2'-deoxycytidine (29). This suggests that the methylation of TIMP3 plays a major role in ependymoma. However, TIMP3 was not differentially methylated in our analysis with respect to patient age and tumor location subgroups.

Our experimental design had some limitations, mainly related to the small number of tumor samples (CNS tumors are relatively rare). As per the power analysis with respect to the methylation study, the actual power was 0.821 and the standard sample number calculated was 15 . Consequently, the number of samples in our article is slightly insufficient to warrant significance. Moreover, the ST group only included one child and all patients in the $\mathrm{PF}$ group were adults. Because posterior ependymomas are more common among children, our patient cohort might not represent the major features present in ependymoma groups. In addition, there was no germline control used for RNA sequencing and CCP because of the shortage of samples. However, this limitation was overcome to a certain degree by comparing the results obtained using three independent molecular analysis methods.

In summary, we analyzed ependymomas from patients, focusing on different tumor grades and locations, as well as on patient age, using next-generation sequencing methods, including methylation array, WES, CCP, and RNA-seq analyses. Although the number of specimens was limited, different combinations of the various conditions allowed sufficient diversity within the cohort to obtain reliable data. This constitutes the first report of next-generation sequencing analysis of ependymomas in Korean patients. Importantly, our findings highlight the need for the application of more accurate diagnostic parameters, other than tumor grade. Furthermore, our study provides major insights into the genetic and epigenetic profiles of intracranial ependymomas, with the potential to impact the development of approaches to improve their prognostic accuracy.

\section{Acknowledgements}

The authors would like to thank Dr Lee Heon Yi (Division of Computer Science, Sookmyung Women's University, Seoul, Republic of Korea) for the help in reanalyzing the data.

\section{Funding}

The present study was supported by the Korea Health Technology R\&D Project, through the Korea Health Industry Development Institute (KHIDI), funded by the Ministry of Health \& Welfare, Republic of Korea (grant no. HI14C1277).

\section{Availability of data and materials}

The datasets used and/or analyzed during the current study are available from the corresponding author on reasonable request.

\section{Authors' contributions}

HJC and HYP have verified the authenticity of all raw data. HJC wrote manuscript drafts and revised the final version of the manuscript. HJC acquired the pathologic and clinical data, and provided data interpretation of the whole-exome sequencing, comprehensive cancer panel, RNA sequencing and DNA methylation. HYP analyzed the data of the whole-exome sequencing, comprehensive cancer panel and RNA sequencing, and KK and HC analyzed the DNA methylation data. SHPaek, SKK, CKP and SHC acquired patients' clinical data, tumor specimens and radiologic data, and provided data interpretation. SHPark conceived the concept, and designed and supervised the study. All authors read and approved the final manuscript.

\section{Ethics approval and consent to participate}

The Institutional Review Board of Seoul National University, College of Medicine and Hospital approved the current retrospective study (using patients' paraffin blocks; approval no. H-1412-137-636). The requirement for written informed consent from patients was waived as the patients donated their cancer tissues to the tissue bank of Seoul National University Hospital (Seoul, Republic of Korea). The current research complied with the recommendations of the Declaration of Helsinki.

\section{Patient consent for publication}

Not applicable.

\section{Competing interests}

The authors declare that they have no competing interests.

\section{References}

1. Louis DN, Ohgaki H, Wiestler OD and Cavenee WK: WHO Classification of Tumours of the Central Nervous System. Revised 4th Edition. International Agency for Research on Cancer. Lyon, pp106-114, 2016.

2. Rogers HA, Kilday J-P, Mayne C, Ward J, Adamowicz-Brice M, Schwalbe EC, Clifford SE, Coyle B and Grundy RG: Supratentorial and spinal pediatric ependymomas display a hypermethylated phenotype which includes the loss of tumor suppressor genes involved in the control of cell growth and death. Acta Neuropathol 123: 711-725, 2012.

3. Kudo H, Oi S, Tamaki N, Nishida Y and Matsumoto S: Ependymoma diagnosed in the first year of life in Japan in collaboration with the International Society for Pediatric Neurosurgery. Childs Nerv Syst 6: 375-378, 1990.

4. Horn B, Heideman R, Geyer R, Pollack I, Packer R, Goldwein J, Tomita T, Schomberg P, Ater J, Luchtman-Jones L, et al: A multi-institutional retrospective study of intracranial ependymoma in children: Identification of risk factors. J Pediatr Hematol Oncol 21: 203-211, 1999.

5. Rubio MP, Correa KM, Ramesh V, MacCollin MM, Jac oby LB, von Deimling A, Gusella JF and Louis DN: Analysis of the neurofibromatosis 2 gene in human ependymomas and astrocytomas. Cancer Res 54: 45-47, 1994.

6. Taylor MD, Poppleton H, Fuller C, Su X, Liu Y, Jensen P, Magdaleno S, Dalton J, Calabrese C, Board J, et al: Radial glia cells are candidate stem cells of ependymoma. Cancer Cell 8: 323-335, 2005.

7. Mack SC, Witt H, Piro RM, Gu L, Zuyderduyn S, Stütz AM, Wang X, Gallo M, Garzia L, Zayne K, et al: Epigenomic alterations define lethal CIMP-positive ependymomas of infancy. Nature 506: 445-450, 2014.

8. Pajtler KW, Witt H, Sill M, Jones DTW, Hovestadt V,Kratochwil F, Wani K, Tatevossian R, Punchihewa C, Johann P, et al: Molecular classification of ependymal tumors across All CNS compartments, histopathological grades, and age groups. Cancer Cell 27: 728-743, 2015. 
9. Ellison DW, Kocak M, Figarella-Branger D, Felice G, Catherine G, Pietsch T, Frappaz D, Massimino M, Grill J, Boyett JM and Grundy RG: Histopathological grading of pediatric ependymoma: Reproducibility and clinical relevance in European trial cohorts. J Negat Results Biomed 10: 7, 2011

10. Du P, Zhang X, Huang CC, Jafari N, Kibbe WA, Hou L and Lin SM: Comparison of Beta-value and M-value methods for quantifying methylation levels by microarray analysis. BMC Bioinformatics 11: 587, 2010

11. Chen J, Bardes EE, Aronow BJ and Jegga AG: ToppGene Suite for gene list enrichment analysis and candidate gene prioritization. Nucleic Acids Res 37: W305-W311, 2009.

12. Agilent Technologies: SureSelect-How it Works. http://www. genomics.agilent.com/article.jsp?pageId=3083. Accessed Nov 10, 2020 .

13. Li H and Durbin R: Fast and accurate short read alignment with Burrows-Wheeler transform. Bioinformatics 25: 1754-1760, 2009.

14. DePristo MA, Banks B, Poplin R, Garimella KV, Maguire JR, Hartl C, Philippakis AA, del Angel G, Rivas MA, Hanna M, et al: A framework for variation discovery and genotyping using next-generation DNA sequencing data. Nat Genet 43: 491-498, 2011.

15. Van der Auwera GA, Carneiro MO, Hartl C, Poplin R, Del Angel G, Levy-Moonshine A, Jordan T, Shakir K, Roazen D, Thibault T, et al: From FastQ data to high confidence variant calls: The Genome Analysis Toolkit best practices pipeline. Curr Protoc Bioinformatics 43: 11.10.1-11.10.33, 2013.

16. Koboldt DC, Zhang Q, Larson DE, Shen D, McLellan MD, Lin L, Miller CA, Mardis ER, Ding L and Wilson RK: VarScan 2: Somatic mutation and copy number alteration discovery in cancer by exome sequencing. Genome Res 22: 568-576, 2012.

17. Wang K, Li M and Hakonarson H: ANNOVAR: Functional annotation of genetic variants from high-throughput sequencing data. Nucleic Acids Res 38: e164, 2010.

18. Trapnell C, Pachter L and Salzberg SL: TopHat: Discovering splice junctions with RNA-Seq. Bioinformatics 25: 1105-1111, 2009.

19. Trapnell C, Williams BA, Pertea G, Mortazavi A, Kwan G, van Baren MJ, Salzberg SL, Wold BJ and Pachter L: Transcript assembly and quantification by RNA-Seq reveals unannotated transcripts and isoform switching during cell differentiation. Nat Biotechnol 28: 511-515, 2010.
20. Koch CM and Wagner W: Epigenetic-aging-signature to determine age in different tissues. Aging (Albany NY) 3: 1018-1027, 2011.

21. Rousseau E, Palm T, Scaravilli F, Ruchoux M-M, FigarellaBranger D, Salmon I, Ellison D, Lacroix C, Chapon F, Mikol J, et al: Trisomy 19 ependymoma, a newly recognized genetico-histological association, including clear cell ependymoma. Mol Cancer 6: 47, 2007.

22. Wu J, Armstrong TS and Gilbert MR: Biology and management of ependymomas. Neuro Oncol 18: 902-913, 2016.

23. Montavon T and Duboule D: Chromatin organization and global regulation of Hox gene clusters. Philos Trans R Soc Lond B Biol Sci 368: 20120367, 2013.

24. Kurscheid S, Pierre Bady P, Sciuscio D, Samarzija I, Shay T, Vassallo I, Criekinge WV, Daniel RT, van den Bent MJ, Marosi C, et al: Chromosome 7 gain and DNA hypermethylation at the HOXA10 locus are associated with expression of a stem cell related HOX-signature in glioblastoma. Genome Biol 16: 16 , 2015.

25. Korshunov A, Neben K, Wrobel G, Tews B, Benner A, Hahn M, Golanov A and Lichter P: Gene expression patterns in ependymomas correlate with tumor location, grade, and patient age. Am J Pathol 163: 1721-1727, 2003.

26. Gu S, Gu W, Shou J, Xiong J, Liu X, Sun B, Yang D and Xie R: The molecular feature of HOX gene family in the intramedullary spinal tumors. Spine (Phila Pa 1976) 42: 291-297, 2017.

27. Korshunov A, Witt H, Hielscher T, Benner A, Remke M, Ryzhova M, Milde T, Bender S, Wittmann A, Schöttler A, et al: Molecular staging of intracranial ependymoma in children and adults. J Clin Oncol 28: 3182-3190, 2010.

28. Parker M, Mohankumar KM, Punchihewa C, Weinlich R, Dalton JD, Li Y, Lee R, Tatevossian RG, Phoenix TN, Thiruvenkatam R, et al: C11orf95-RELA fusions drive oncogenic NF-kappaB signalling in ependymoma. Nature 506: 451-455, 2014.

29. Karakoula K, Jacques TS, Phipps KP, Harkness W, Thompson D, Harding BN, Darling JL and Warr TJ: Epigenetic genome-wide analysis identifies BEX1 as a candidate tumour suppressor gene in paediatric intracranial ependymoma. Cancer Lett 346: 34-44, 2014. 